\title{
Study on Hydrogen Content in Cord Steel by Carrier gas hot extraction
}

\author{
Qiang $\mathrm{Li}^{1}$, Ke Zhu ${ }^{1}$, Bei Huang ${ }^{1}$, Xide $\mathrm{Li}^{2}$, Xuehai Qian ${ }^{2}$, and Jianmin Zeng ${ }^{1, *}$ \\ ${ }^{1}$ College of Resources, Environment and Materials, Guangxi University, Nanning China \\ ${ }^{2}$ Research Institute of Guangxi Liuzhou Iron and Steel Group Co., Ltd., Liuzhou China
}

\begin{abstract}
Cord steel has very strict requirements on the content of impurities. Hydrogen is a harmful gas element in steel and is the cause of hydrogen embrittlement. In this work, the Carrier gas hot extraction method is used to measure the hydrogen content of 77A steel. It was found that the measured value of hydrogen content was related to cooling capability of the mold. It is found that with the increasing of cooling speed of the mold, the hydrogen content increases. The measured hydrogen content can roughly reflect the hydrogen content in the liquid alloy by using the cast iron mold with faster cooling speed.
\end{abstract}

\section{Introduction}

Steel cord can be used for rubber product skeleton, high-strength wire, steel bar for reinforced concrete structure, railway switch, bridge component, etc. [1-2]. As cord wire rod is drawn into $\Phi 0.15 \sim 0.38 \mathrm{~mm}$ thin wire, it requires high strength and good toughness. Therefore, it is required that the steel must have extremely high cleanliness. Hydrogen is a harmful element in steel. In the process of steel solidification, if hydrogen dissolved in molten steel is not released in time, it will diffuse to the vicinity of defects in the steel after solidification[4-6]. At room temperature, atomic hydrogen will combine into molecular hydrogen at the defects and accumulate continuously, thus generating huge internal pressure and cracking the metal. Therefore, the determination of hydrogen content in steel is of great significance for controlling the metallurgical quality of molten steel. Due to the high temperature of molten steel, on-line measurement of hydrogen is very difficult. Therefore, Carrier gas hot extraction will be used for obtaining hydrogen content data in 77A steel.

\section{Experimental}

\subsection{Equipment}

The diffusion hydrogen analyzer was employed to conduct the experiment, as shown in figure 1 . The measurement method is Carrier gas hot extraction. The main components of the analyzer are a heating furnace loaded with quartz tubes, a thermal conductivity detector, a gas calibration unit, a diaphragm pump, etc. Figure 1 is a schematic of the equipment. Diffuse hydrogen was determined by carrier gas thermal extraction method. Nitrogen with purity of $99.999 \%$ was used as carrier gas, and the sample was put into the heating zone for heating. As the heating process progresses, the hydrogen in the sample is released and enters the thermal conductivity detector along with the carrier gas flow. Due to the large difference in thermal conductivity between nitrogen and hydrogen, there is an obvious difference in heat conductivity, which affects the resistance value of the heat sensitive element. Moreover, because the heat sensitive element is connected to the Wheatstone bridge, the difference in thermal conductivity between the two gases will directly affect the output of the voltage signal. The magnitude of the voltage signal is proportional to the hydrogen content. The total voltage value can be obtained by integrating the voltage signal-time curve, and the hydrogen content of the sample can be calculated.

\subsection{Sample preparation}

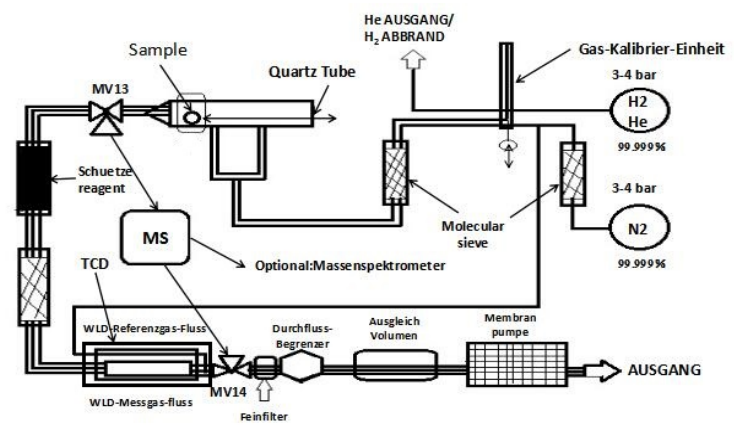

Fig. 1. Schematic diagram of hydrogen diffusion analyzer

*Corresponding Author: zjmg@gxu.edu.cn 
The experimental material is 77A steel, the main composition is C $0.78 \%$, Si $0.26 \%$, Mn $0.45 \%$. After melting in medium frequency induction furnace, it was poured into insulate refractory mold, sand mold and cast iron mold, respectively. The cast sample is $10 \mathrm{~mm}$ long and $5 \mathrm{~mm}$ width. The oxide scale on the sample surface was carefully removed. In order to prevent water vapor in the atmosphere from adsorbing on the surface of the sample, the prepared sample must be tested for diffused hydrogen as soon as possible.

\section{Result and analysis}

The hydrogen evolution curves of the cast samples at different cooling rates is shown in figure 2. It can be seen from the figure that the magnitude of the evolution peak is related to the cooling rate of the mold. The higher the cooling rate, the higher the precipitation peak. Secondly, there will be an incubation period at the beginning, that is, the period from the beginning of heating to the time before hydrogen precipitation in the sample. From the precipitation time of diffused hydrogen, it can be seen that the incubation period is related to the properties of the material. At the same time, the oxidation film on the surface of the sample will also influence the diffusion of hydrogen, thus prolonging the incubation period.

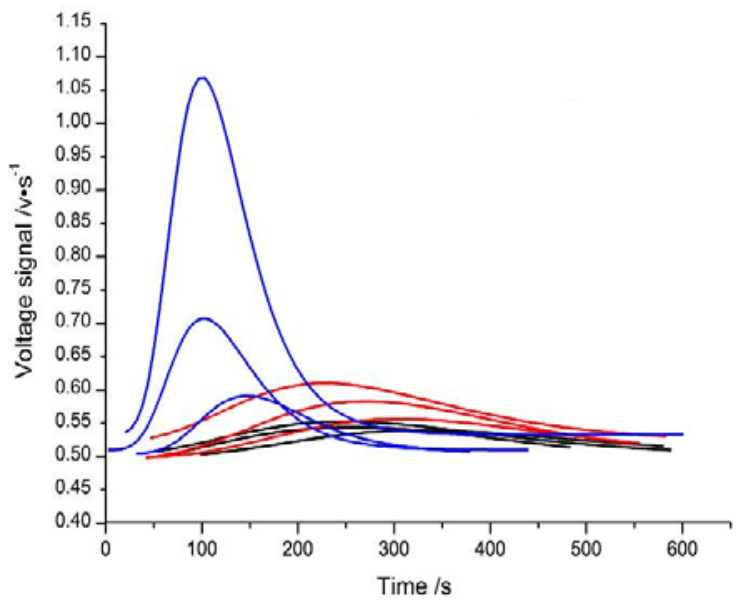

Fig. 2. Voltage signal with different mold materials

The reason for the incubation period is analyzed. The hydrogen atoms or molecules of the sample are "fixed" in metals in various ways, so the diffusion of hydrogen atoms from the sample must overcome the "fixed" binding force. The binding force here refers to the energy barrier that hydrogen atoms need to overcome to diffuse out of the sample. From the perspective of dynamics, the migration of hydrogen atoms between lattices will cause distortion of the surrounding lattices, so atoms must obtain activation energy to ensure smooth transition. The process of heating the sample is the process of hydrogen atoms obtaining energy. When the heating temperature is large enough or long enough, hydrogen atoms are provided with energy to break free from bondage, so that hydrogen atoms can obtain enough energy to overcome the energy barrier to do work, and then migrate between lattices and finally escape to the metal surface.

\subsection{The data processing}

The relationship between hydrogen content and the voltage can be expressed below:

$$
c=k u
$$

where, $c$ is the hydrogen Content in Sample and $\mathrm{u}$ is the voltage of thermal conductivity detector. The hydrogen evolution curve is considered to conform to the following equation:

$$
\begin{gathered}
u=u_{0}+A \exp (-\exp (-z)-z+1) \\
z=\frac{t-t_{c}}{w}
\end{gathered}
$$

Wherein $u_{0}, t c, A$ and $w$ are constants, and their values vary according to different curves. Fitting the equation with the voltage signal-time curve can obtain the corresponding parameter values. integrating the curve, the hydrogen content $C$ in the sample can be obtained according to the following equation:

$$
C=\int_{0}^{t} c d t=k \int_{0}^{t} u d t
$$

that is:

$$
C=k \int_{0}^{t}\left[u_{0}+A \exp (-\exp (-z)-z+1)\right] d t
$$

Table 1 shows the analysis results of hydrogen content in samples at different cooling rates.

Table 1. Hydrogen content at different cast mold

\begin{tabular}{|c|c|c|c|}
\hline \multirow{2}{*}{ Mold material } & \multicolumn{3}{|c|}{ Hydrogen content (ml/100g metal) } \\
\hline \multirow{2}{*}{$\begin{array}{c}\text { Insulate } \\
\text { refractory }\end{array}$} & 0.02 & 0.06 & 0.03 \\
\cline { 2 - 4 } & 0.06 & 0.11 & 0.08 \\
\hline \multirow{2}{*}{$\begin{array}{c}\text { Dry sand } \\
\text { mold }\end{array}$} & 0.31 & 0.42 & 0.33 \\
\cline { 2 - 4 } & 0.19 & 0.26 & 0.42 \\
\hline \multirow{2}{*}{ Grey cast iron } & 0.46 & 0.33 & 0.36 \\
\cline { 2 - 4 } & 0.38 & 0.36 & 0.29 \\
\hline
\end{tabular}

It can be seen that the hydrogen content increases in turn according to the cooling mode of insulate refractory mold, sand mold and cast iron mold, with the average hydrogen content of $0.06 \mathrm{ml} / 100 \mathrm{~g}, 0.36 \mathrm{ml} / 100 \mathrm{~g}$ and $0.73 \mathrm{ml} / 100 \mathrm{~g}$, respectively. The analysis shows that the three different cooling media have different thermal conductivity and therefore different cooling rates, resulting in different solidification times of metals. When the solidification time is very short, the hydrogen in the sample does not have time to 
diffuse, thus remaining in the melt. The heat storage coefficient of the three molds gradually increases, so the solidification time increases continuously, and the hydrogen in the sample does not have enough time to diffuse from the sample, resulting in increase in the hydrogen contents.

\section{Conclusion}

In this work, hydrogen content of 77A cord steel was measured with hydrogen diffusion apparatus. Diffuse hydrogen was determined by carrier gas thermal extraction method. The sample was put into the heating zone for heating. As the heating process progresses, the hydrogen in the sample is released and enters the thermal conductivity detector along with the carrier gas flow. Due to the large difference in thermal conductivity between nitrogen and hydrogen, there is an obvious difference in heat conductivity, which affects the resistance value of the heat sensitive element. Moreover, because the heat sensitive element is connected to the Wheatstone bridge, the difference in thermal conductivity between the two gases will directly affect the output of the voltage signal. The voltage signal is proportional to the hydrogen content, the hydrogen content of the sample can be calculated.

It is found that with the increasing of cooling rate, the solubility of hydrogen in the material increases, thus resulting in the increase in hydrogen content. The measured hydrogen content can also roughly reflect the hydrogen content in the liquid alloy by using the cast iron mold with faster cooling speed.

\section{Acknowledgement}

This work has been financially supported by Guangxi major project (AA18242013) of Science and Technology and by the support of the project in the collaborative innovation of ecological aluminum industry in Guangxi.

\section{References}

1. Parusov, V.V., Derevyanchenko, I.V., Sychkov, A.B., et al. Ensuring High Quality Indices for the Wire Rod Used to Make Metal Cord. Metallurgist 49, 439-448 (2005).

2. Lee, S.K., Ko, D.C. and Kim, B.M., Pass Schedule of Wire Drawing Process to Prevent Delamination for High Strength Steel Cord Wire. Materials and Design 30, 2919-2927 (2009).

3. Qiang Li, Ke Zhu, Xuehai Qian, Xide Li, Ni Wu, Zhuowei Meng, Jianmin Zeng, Research Progress of Cord Steel Production Technology, Metallurgical Engineering 6, 251-259 (2019).

4. H. K. D. H. Bhadeshia, Prevention of Hydrogen Embrittlement in Steels, ISIJ International 56, 24-36 (2016).

5. Sandeep kumar Dwivedi. Hydrogen embrittlement in different materials: A review. International Journal of Hydrogen Energy 43, 21603-21616 (2018).

6. Dan Eliezera, Ravit Silverstein. Recent Studies of Hydrogen Embrittlement in Structural Materials. Procedia Structural Integrity 13, 2233-2238 (2018). 